\title{
Reabilitação auditiva na criança: adesão ao tratamento e ao uso do aparelho de amplificação sonora individual
}

\author{
Hearing rehabilitation in children: adhesion to treatment and use \\ of hearing aids
}

Juliana Habiro de Souza Miguel'1, Beatriz Cavalcanti de Albuquerque Caiuby Novaes²

\begin{abstract}
RESUMO
Objetivo: Discutir a adesão das famílias na fase inicial de intervenção quanto ao uso do Aparelho de Amplificação Sonora (AASI), a participação no processo, e sua relação com desempenho auditivo e de linguagem das crianças com deficiência auditiva. Métodos: Estudo realizado com pais e/ou responsáveis de 16 crianças deficientes auditivas em processo de ADAPTI (Adaptação de AASI e Terapia Inicial) no ano de 2009 e que estavam regularmente inscritas no Serviço de Saúde Auditiva Centro Audição na Criança - CeAC. Os pais foram divididos em três grupos: Pré-determinado - PD (famílias participantes da terapia fonoaudiológica e do Grupo de Adesão Familiar - GrAF, do primeiro ao último dia de atendimento estabelecido); Fluxo contínuo - FC (famílias que iniciavam a participação no GrAF a partir do momento em que chegavam ao serviço de saúde para a adaptação de AASI, sem início estabelecido) e Controle - C (famílias que participaram somente da terapia fonoaudiológica, mas não do GrAF). Resultados: Foi constatada a efetividade da adesão dos pais ao tratamento dos filhos, sendo registrado o comparecimento em mais de $66 \%$ dos atendimentos propostos (terapia fonoaudiológica e/ou GrAF). Na análise estatística dos agrupamentos de crianças, foi possível a formação de três diferentes grupos no que se refere ao uso do datalog (horas/dia), envolvimento familiar e grau de perda auditiva. Conclusão: O uso sistemático de AASI foi a única variável com forte relação com habilidades auditivas e de linguagem. O grau de perda auditiva e a idade de início de amplificação não explicaram o desenvolvimento das crianças do estudo.
\end{abstract}

Descritores: Família; Reabilitação; Perda auditiva; Auxiliares de audição; Desenvolvimento da Linguagem

\begin{abstract}
Purpose: Early diagnosis of hearing loss in children, as well as fast intervention, have been shown to be determining factors for hearing and language development, with important implications for the social inclusion process and communicative performance. The present study aims to analyze alternatives that guarantee adhesion to use of Hearing Aids and greater family participation in the initial steps of the intervention. Methods: The study comprehended parents/caregivers of 16 hearing impaired children who attended the process of ADAPTI (Hearing Aid Fitting and Initial Therapy) during one year, who had formally applied for the Hearing Care Service. The parents were divided in three groups: Previously Determined (Families that attend both the speech therapy and the Family Adhesion Group (GrAF) from the first day set for the beginning of sessions, to the last day); Continuous Flow (Families that started attending the group from the moment they arrived for the ADAPTI); and Control (Families that only attended speech therapy, but not the GrAF). Results: The study verified the effectiveness of parent adhesion to their children's treatment with more than $66 \%$ of attendance in the proposed activities (therapeutic intervention and/or GrAF). The statistical analysis allowed the composition of three children groups regarding the datalog usage (hours a day), family participation and degree of hearing loss. Conclusion: The systematic use of hearing aids was the only variable closely related to hearing and language skills. Degree of hearing loss and age at the beginning of amplification have not explained the development of the children in the study.
\end{abstract}

Keywords: Family; Rehabilitation; Hearing loss; Hearing aids; Language development

Trabalho desenvolvido no Programa de Estudos Pós-Graduados em Fonoaudiologia, Pontifícia Universidade Católica de São Paulo - PUC-SP - São Paulo (SP), Brasil, com bolsa concedida pela Coordenação de Aperfeiçoamento de Pessoal de Nível Superior (CAPES).

(1) Departamento de Otorrinolaringologia, Irmandade da Santa Casa de Misericórdia de São Paulo - São Paulo (SP), Brasil.

(2) Programa de Pós-graduação em Distúrbios da Comunicação Humana, Pontifícia Universidade Católica de São Paulo - PUC-SP - São Paulo (SP), Brasil.

Conflito de interesses: Não

Contribuição dos autores: JHSM pesquisador principal, elaboração da pesquisa, elaboração do cronograma, levantamento da literatura, coleta e análise dos dados, redação do artigo, submissão e trâmites do artigo; $B C A C N$ orientadora, elaboração da pesquisa, elaboração do cronograma, análise dos dados, correção da redação do artigo, aprovação da versão final.

Endereço para correspondência: Juliana Habiro de Souza Miguel. R. Camarajé 88/143, Mandaqui, São Paulo (SP), Brasil, CEP: 02416-060.

E-mail: juhabiro@yahoo.com.br

Recebido em: 31/7/2012; Aceito em: 29/7/2013 


\section{INTRODUÇÃO}

O diagnóstico precoce da deficiência auditiva em crianças, e o início da intervenção o mais cedo possível, são apontados como determinantes no desenvolvimento auditivo e de linguagem, com importantes implicações no processo de inclusão social e desempenho comunicativo.

Muitos autores referem que, para um melhor prognóstico, o diagnóstico e a intervenção devem ocorrer antes dos 6 meses de vida, garantindo, assim, o melhor aproveitamento do potencial auditivo da criança. Além disso, interferem nesse prognóstico, o uso efetivo do aparelho auditivo ou Implante Coclear, as expectativas dos familiares, o grau de envolvimento com o tratamento e os aspectos relativos às condições socioeconômicas e culturais ${ }^{(1-7)}$.

O trabalho fonoaudiológico que visa à aquisição da oralidade tem sido um dos principais eixos de propostas clínicas para a criança com deficiência auditiva. Implica considerar suas particularidades na constituição do desenvolvimento social, emocional, psíquico e cognitivo, além de reordenar as situações de interação, favorecendo, assim, o modo de comunicação desse grupo de pacientes ${ }^{(4,8-10)}$.

Porém, para que esse ideal seja alcançado, é necessário que os pais das crianças com deficiência auditiva entendam o que buscam no Serviço de Saúde Auditiva, para fazer suas escolhas com clareza e discernimento, compreendendo as potencialidades de seus filhos e ajustando suas expectativas no decorrer desse processo.

Os contatos da família com o fonoaudiólogo no processo de adaptação de Aparelho de Amplificação Sonora Individual (AASI) servem também para a explicitação dos objetivos do tratamento e das opções clínicas e educacionais disponíveis na comunidade. No âmbito da saúde auditiva, é fundamental esclarecer, ainda, a função do uso de amplificação para o desenvolvimento auditivo e de linguagem oral.

A intervenção envolve diversos profissionais com capacidade de absorver e atender com cautela essa demanda de pacientes que precisam de atenção especial. Desta forma, é importante que o profissional da saúde tenha condições de perceber as mudanças que eventualmente ocorram no contexto familiar, as angústias diante do problema e as possíveis negações, para que possa contribuir no processo de reorganização da família diante do diagnóstico, acompanhando o desenvolvimento da criança $^{(11)}$.

O Centro Audição na Criança (CeAC), da Divisão de Educação e Reabilitação dos Distúrbios da Comunicação (DERDIC) e parte da estrutura da Pontifícia Universidade Católica de São Paulo (PUC-SP), é um serviço de alta complexidade, credenciado pelo Serviço Único de Saúde (SUS), que oferece atendimento a crianças com suspeita de deficiência auditiva, ou com o diagnóstico confirmado abaixo dos 3 anos de idade. São oferecidos: diagnóstico, seleção e indicação de AASI, terapia fonoaudiológica, acompanhamento e orientações às famílias. O CeAC também oferece terapia para uma parte da demanda que é criada pelo seu próprio serviço de diagnóstico, sendo a outra parte encaminhada para serviços da rede municipal, ou para outros locais de atendimento próximos às residências das famílias. Isso implica a necessidade de se realizar procedimentos de avaliação sistemática da audição, linguagem e envolvimento familiar nos acompanhamentos, visando garantir a qualidade dos atendimentos de todas as crianças, dentro e fora do centro, que é o responsável pelo seguimento dos casos.

Quando se conclui o diagnóstico e é confirmada a deficiência auditiva, paralelamente à seleção do AASI, realiza-se o processo de adaptação do aparelho, já envolvendo as etapas iniciais do processo terapêutico, que têm como objetivo, além do acolhimento e orientações iniciais aos pais, realizar a adaptação e iniciar terapia de linguagem, com ênfase na participação da família, compondo a etapa denominada processo ADAPTI.

Como parte do processo terapêutico inicial, ocorre, semanalmente, o Grupo de Adesão Familiar (GrAF), cujo objetivo é propiciar ações de diferentes modalidades com todos os pais das crianças. O GrAF reúne pais iniciantes para discutir o potencial auditivo da criança, as modalidades de intervenção possíveis para cada uma delas e o encaminhamento para programas apropriados de intervenção. Esse período dura, em média, quatro meses, dependendo da disponibilidade da família e do processo de contrarreferência para centros mais próximos à residência.

Em razão da importância da família em todas as etapas de intervenção com a criança para atingir melhor prognóstico, o GrAF tem como meta contribuir para aproximar os pais da reabilitação auditiva de seus filhos, buscando desenvolver um conjunto de ações de diferentes modalidades que os envolva na etapa inicial do tratamento. Tais ações integram e iniciam o processo terapêutico que, nessa etapa, é realizado por meio de enquadre centrado nos pais ou responsáveis.

Historicamente, tem-se observado que algumas famílias se envolvem rapidamente no processo, alcançam o uso consistente do AASI e posicionam-se em relação ao tratamento. Outras, parecem contentar-se com a vaga no serviço, porém não sabem o que esperar do uso do dispositivo e, muitas vezes, embora frequentando regularmente as terapias, as crianças não o utilizam em casa.

Diante desse quadro, o presente estudo buscou analisar alternativas que possam garantir a adesão ao uso do AASI e maior envolvimento da família nas etapas iniciais da intervenção.

De fato, são necessárias propostas de ações no âmbito da intervenção em Serviços de Saúde Auditiva que garantam a adesão ao tratamento, tanto no que se refere ao envolvimento familiar - disponibilidade para a participação - quanto à utilização sistemática e consistente do AASI. Além disso, relações entre desempenho auditivo e de linguagem e monitoramento da adesão da família podem contribuir para melhor compreensão do processo terapêutico e, consequentemente, para melhores resultados na Saúde Auditiva. 
O objetivo deste estudo foi discutir a adesão das famílias de crianças com deficiência auditiva na fase inicial de intervenção, quanto ao uso do aparelho de amplificação sonora individual, a participação no processo e sua relação com desempenho auditivo e de linguagem.

\section{MÉTODOS}

Foi realizado um estudo qualitativo descritivo com os pais e/ou responsáveis e terapeutas de 16 crianças deficientes auditivas que iniciaram o atendimento no ADAPTI (Adaptação de AASI e Terapia Inicial) e que estavam regularmente inscritas no CeAC. Obedecendo aos princípios éticos, todos os participantes assinaram o Termo de Consentimento Livre e Esclarecido. Este estudo foi aprovado pelo Comitê de Ética em Pesquisa da Pontifícia Universidade Católica de São Paulo (PUC), sob parecer 292/2008.

Os participantes do estudo foram divididos em três tipos de modalidades, relativas à participação no Grupo de Adesão Familiar - GrAF e tipo de enquadre:

- Pré-determinado (PD): enquadre composto por seis famílias de crianças atendidas no ADAPTI. Essas famílias participaram tanto da terapia fonoaudiológica, quanto do GrAF, no primeiro semestre de 2009, do primeiro ao último dia de atendimento estabelecido, com previsão de doze encontros. Nesse grupo não ocorreram entradas de familiares após o início do atendimento, nem saídas antes do tempo estabelecido e acordado por todos os participantes.

- Fluxo contínuo (FC): enquadre composto por seis famílias que participaram do GrAF no segundo semestre de 2009, a partir do momento em que chegavam ao CeAC e iniciavam o ADAPTI. Foi permitida a participação imediata no GrAF já em andamento. Estavam previstos quinze encontros.

- Controle (C): enquadre composto por quatro famílias do ADAPTI que participaram da terapia fonoaudiológica, mas não do GrAF. Foram realizadas as 16 sessões previstas, com acolhimento e orientação aos pais.

A inclusão dos sujeitos obedeceu aos seguintes critérios: serem membros da família da criança deficiente auditiva, acompanhando-a regularmente ao atendimento fonoaudiológico realizado no CeAC; a criança deveria estar utilizando AASI há, no máximo, seis meses, com agendamento no ADAPTI; disponibilidade dos pais ou responsáveis para frequentar as atividades propostas nos horários estabelecidos e concordância na participação do estudo.

Com o objetivo de avaliar o envolvimento familiar no processo terapêutico da criança deficiente auditiva, alguns instrumentos foram utilizados:

- Roteiro de Entrevista ${ }^{(12)}$ : perguntas que levam os pais a identificar as atitudes de comunicação da criança, a participação desses pais no programa de intervenção, as orientações recebidas para lidar com a criança e as expectativas em relação ao desenvolvimento do seu filho com deficiência auditiva;
- Escala de Avaliação de Envolvimento Familiar ${ }^{(1)}$ : escala denominada Family Involvement Rating, composta por cinco itens: 1 - participação limitada; 2 - participação abaixo da média; 3 - participação mediana; 4 - boa participação e 5 - participação ideal. As famílias foram classificadas pelos terapeutas, que tiveram extenso contato com os pais e com a criança;

- MUSS $^{(13)}$ : escala em forma de questionário, com a finalidade auxiliar na avaliação da linguagem oral/habilidades de produção de fala de crianças em situações de vida diária;

- IT MAIS ${ }^{(14)}$ : tem como objetivo avaliar comportamentos auditivos de crianças em situações de vida diária, refletindo sobre mudanças na vocalização, integradas ao uso do dispositivo eletrônico, atenção/alerta para os diversos sons ambientais e atribuição de significado aos sons;

- Atividades de sensibilização cultural ${ }^{(12)}$ : utilizadas como disparadoras nos GrAF, visando deixar os pais mais à vontade para falar de suas próprias questões individuais e, assim, trazer as questões relativas aos seus filhos. As atividades em si não foram avaliadas e descritas após o GrAF, pois tinham como único objetivo serem disparadoras de conversas e discussões quanto às questões auditivas e de linguagem das crianças atendidas;

- Avaliação do uso efetivo do AASI: registro do uso médio de horas diárias.

A partir dos dados obtidos nos diferentes procedimentos e instrumentos utilizados nesta pesquisa, a análise foi feita considerando os seguintes recortes:

1. Caracterização dos familiares pelos grupos PD, FC e C quanto a gênero, idade, nível de escolaridade e idade; avaliação das presenças e faltas das famílias nos atendimentos (terapêutico e GrAF); descrição das características audiológicas das crianças estudadas com relação aos seguintes aspectos: idade do início da seleção de AASI (meses); grau da perda auditiva; dispositivo eletrônico utilizado e proposta terapêutica após o término do grupo. A frequência de uso diário do AASI foi determinada no momento do acompanhamento periódico do serviço, por meio do registro do Datalog, nas crianças em que o dispositivo estava disponível.

2. Estabelecimento de grupos de crianças caracterizados quanto a IT MAIS, MUSS, Datalog horas/dia, Envolvimento Familiar e Grau de Perda Auditiva, por meio da elaboração de tabelas com valores de estatísticas descritivas dessas variáveis, por agrupamentos formados. Também foram elaboradas tabelas com as frequências e porcentagens das categorias de quem participou do GrAF. A condição estabelecida foi a de que os grupos fossem homogêneos internamente e heterogêneos entre si, quanto às variáveis IT MAIS e MUSS. A técnica adotada foi a da média das distâncias. A distância entre os indivíduos foi medida por meio da distância Euclidiana, pela aplicação da técnica de análise de agrupamentos ${ }^{(15)}$. 
3. Para o estudo estatístico, as famílias C1 e PD3 foram excluídas, pois a $\mathrm{C} 1$ já tinha realizado a cirurgia do Implante Coclear no momento em que foi solicitada a responder os protocolos IT MAIS e MUSS, e a PD3 estava sendo reintegrada ao atendimento após um longo período de faltas. Portanto, os dados de ambas as famílias foram utilizados somente para avaliar a sua participação no GrAF e não na análise estatística conjunta com os outros sujeitos da pesquisa. Isso se justifica, pois os resultados causariam uma discrepância muito grande para análise fidedigna da amostra.

As idades das crianças, no momento da análise, foram arredondadas para 15 dias ou menos para o mês anterior e para 15 dias ou mais para o mês posterior (por exemplo: 12 meses e 13 dias $=12$ meses ou 12 meses e 17 dias $=13$ meses).

\section{RESULTADOS}

No grupo Pré-determinado (PD) participaram do GrAF 8 familiares de 6 crianças, sendo 5 mães (63\%), 2 pais (25\%) e 1 avó (12\%); no grupo Fluxo contínuo (FC) participaram do GrAF 9 familiares, sendo 6 mães (67\%) e 3 pais (33\%) e no grupo Controle (C) não aconteceu o $\mathrm{GrAF}$, apenas as sessões terapêuticas. A idade do início do uso do AASI pelas crianças atendidas no ADAPTI variou de 24 dias a 47 meses

A frequência dos pais, no ADAPTI, foi avaliada com o intuito de observar a adesão ao tratamento dos filhos. Neste estudo, observou-se a adesão dos pais ao tratamento (GrAF e terapia). Nove dos $16(56,25 \%)$ participantes compareceram a $66,6 \%$ ou mais dos GrAF realizados e 5 dos 12 (41,66\%) participantes compareceram a $72,7 \%$ ou mais das terapias realizadas. Todas as famílias foram orientadas a permanecer no serviço por duas horas, para participar, tanto dos atendimentos terapêuticos, quanto dos GrAF. O Dendrograma obtido na análise de agrupamentos, sugerindo a existência de três grupos homogêneos quanto ao IT MAIS e MUSS, simultaneamente, é apresentado na Figura 1.

Esse tipo de análise agrupa sujeitos com maior probabilidade de desempenhos semelhantes nos aspectos analisados. A partir dessa análise as famílias foram organizadas em três grupos:

Grupo 1: Famílias FC5, FC6, FC3, C2 e PD2, cujas crianças apresentaram tendência a menores valores de IT MAIS e MUSS. Além disso, foram constatadas semelhanças em relação à presença superior a 50\% nos atendimentos terapêuticos e às diferenças das perdas auditivas das crianças.

Grupo 2: Famílias PD6, C4, FC4, PD1, PD5, cujas crianças situaram-se em um grupo intermediário, no que se refere aos valores de IT MAIS e MUSS e apresentaram média (500 a 2 $\mathrm{kHZ}$ ) de perda auditiva maior que 80dBNA.

Grupo 3: Famílias C3, PD4, FC2, FC1, cujas crianças tenderam a apresentar maiores valores de IT MAIS e MUSS. Além disso, assemelhavam-se nas médias (500 a 2 kHZ) das perdas auditivas, que estavam entre 55 e $90 \mathrm{dBNA}$

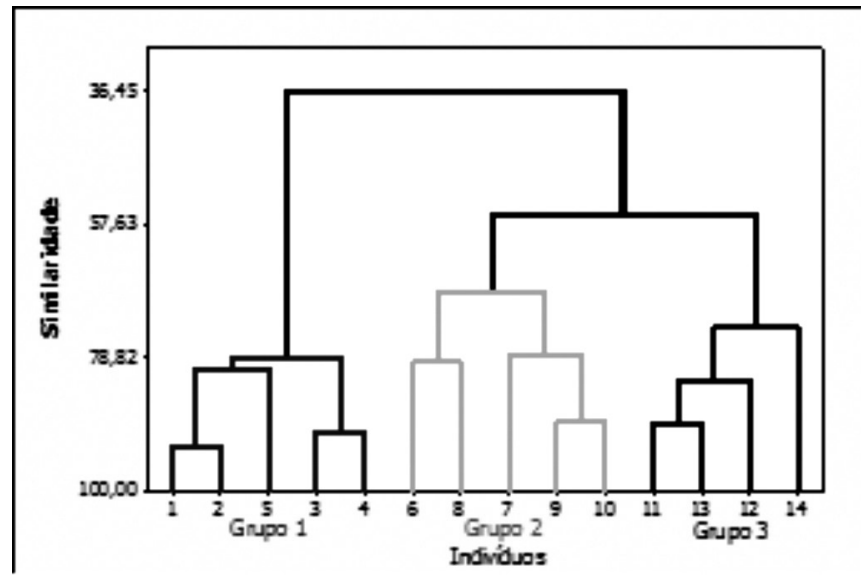

Legenda: Grupo 1 (Famílias: FC5: Vit- FC6: Vi - FC3: Ir - C2: Br, PD2: Ma); Grupo 2 (Famílias - PD6: He; C4: St, FC4: Lu; PD1: Ga; PD5: Ma); Grupo 3 (Famílias C3: Fr - PD4: Ra - FC2: Ro - FC1: Ed)

Figura 1. Dendrograma obtido na análise de agrupamentos com as variáveis IT MAIS e MUSS

As médias e medianas das duas variáveis observadas foram menores no grupo 1 do que nos demais grupos. No grupo 3 foram observadas as maiores médias e medianas. Assim, pode-se dizer que as crianças do grupo 1 tenderam a apresentar os menores valores de IT MAIS e MUSS e as do grupo 3, os maiores valores. Nesse aspecto, o grupo 2 mostrou-se um grupo intermediário. Esse resultado pode ser visualizado no diagrama de dispersão de IT MAIS e MUSS, sendo que os pontos foram identificados de acordo com os grupos (cada ponto corresponde a uma criança) (Figura 2).

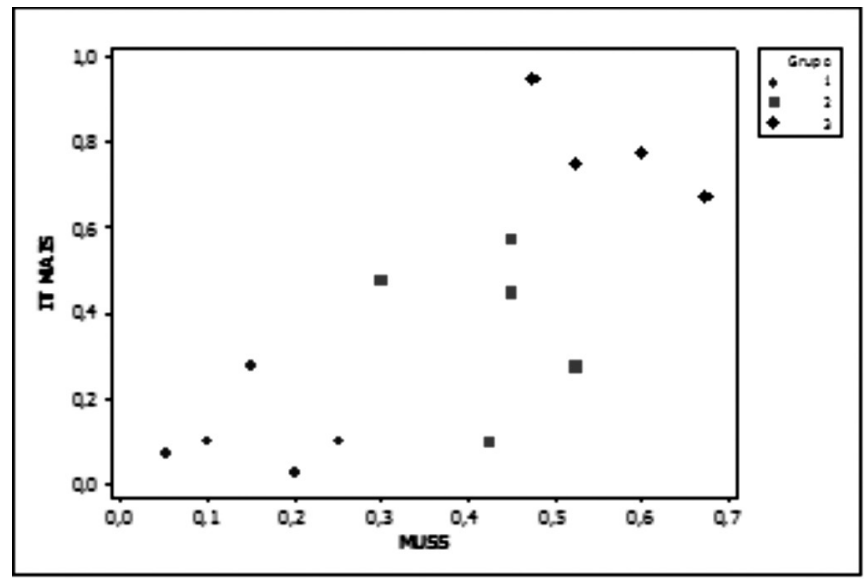

Figura 2. Diagrama de dispersão de IT MAIS e MUSS

Os valores de estatísticas descritivas para Grau de Perda Auditiva, Envolvimento Familiar e Datalog (horas/dia), respectivamente, são apresentados nas Tabelas 1, 2 e 3. Os valores individuais e médios dessas variáveis por grupo estão representados nas Figuras 3, 4 e 5.

Observa-se na Tabela 1 e na Figura 3 que os indivíduos do grupo 3 apresentaram, em média, menor grau de perda auditiva do que os dos grupos 1 e 2 . 
Tabela 1. Estatísticas descritivas para grau de perda, por grupo

\begin{tabular}{|c|c|c|c|c|c|c|}
\hline Grupo & $\mathrm{n}$ & Média & Desvio-padrão & Mínimo & Mediana & Máximo \\
\hline 1 & 5 & 94 & 16,4 & 75 & 95 & 110 \\
\hline 2 & 5 & 97 & 9,7 & 85 & 100 & 110 \\
\hline 3 & 4 & 70 & 14,7 & 55 & 67,5 & 90 \\
\hline Total & 14 & 88,2 & 17,5 & 55 & 90 & 110 \\
\hline
\end{tabular}

Tabela 2. Estatísticas descritivas para envolvimento familiar, por grupo

\begin{tabular}{lcccccc}
\hline Grupo & $\mathrm{n}$ & Média & Desvio-padrão & Mínimo & Mediana & Máximo \\
\hline 1 & 5 & 3,0 & 1,0 & 2 & 3 & 3 \\
2 & 5 & 3,4 & 0,9 & 3 & 3 & 3,5 \\
3 & 4 & 3,5 & 0,6 & 2 & 3 & 5 \\
\hline Total & 14 & 3,3 & 0,8 & 2 & 5 \\
\hline
\end{tabular}

Tabela 3. Estatísticas descritivas para Datalog horas/dia, por grupo

\begin{tabular}{lcccccc}
\hline Grupo & $\mathrm{n}$ & Média & Desvio-padrão & Mínimo & Mediana & Máximo \\
\hline 1 & 3 & 3,0 & 1,0 & 2 & 3 & 4 \\
2 & 3 & 3,7 & 0,6 & 3 & 4 & 6,5 \\
3 & 4 & 5,8 & 2,6 & 2 & 4 & 8 \\
\hline Total & 10 & 4,3 & 2,1 & 2 & & 4 \\
\hline
\end{tabular}

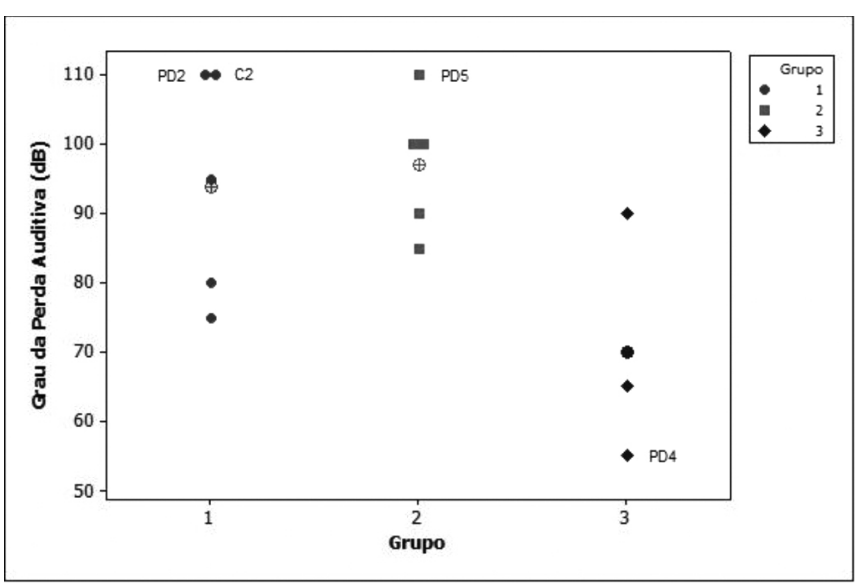

Figura 3. Valores individuais e médios do grau de perda auditiva por grupo

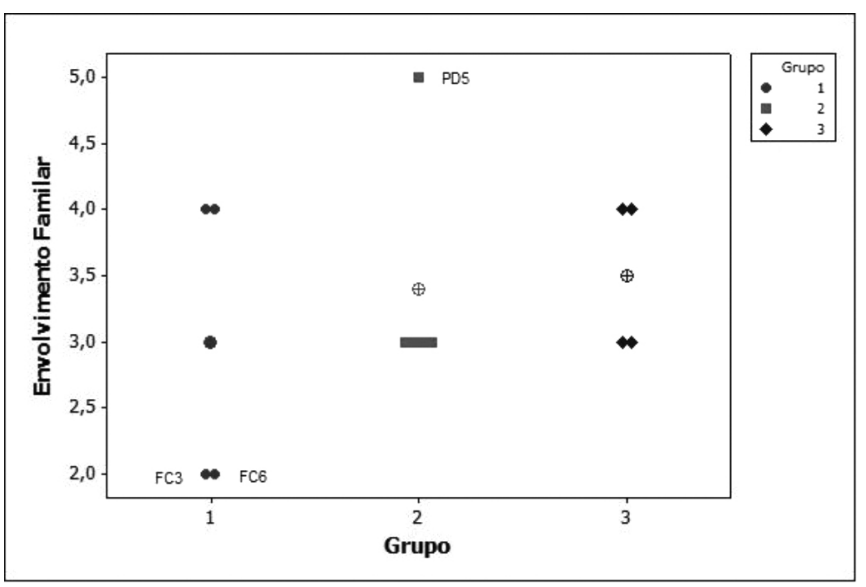

Figura 4. Valores individuais e médios do envolvimento familiar por grupo

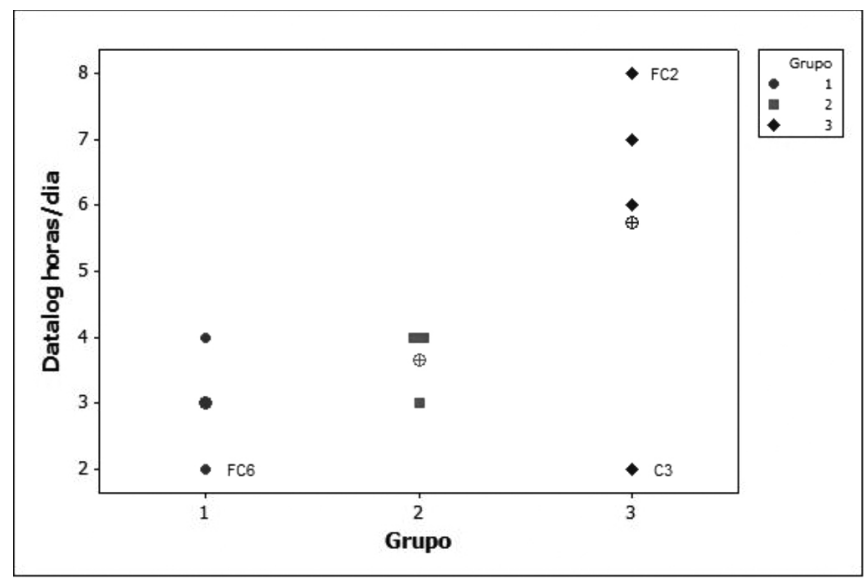

Figura 5. Valores individuais e médios de Datalog horas/dia por grupo

Em relação ao grau de perda auditiva, foi possível verificar que essa não foi uma variável que justificasse a formação dos agrupamentos.

As crianças das famílias PD2 e C2 e PD5 apresentavam perda auditiva de 110 dBNA (média de 500 a $2 \mathrm{kHZ}$ ), sendo respectivamente representadas nos grupos 1 e 2 . Aquelas com o mesmo grau de perda auditiva foram reunidas em diferentes grupos em relação às respostas no IT MAIS e MUSS, devido às expectativas e possibilidades auditivas e de linguagem apresentadas por cada.

A criança da família PD2 não pôde se submeter à cirurgia do IC, pois apresentava má formação de orelha interna, além das cócleas ossificadas bilateralmente. Já as crianças das famílias C2 e PD5 estavam em avaliação para a realização da cirurgia. 
Assim, mesmo com grau de perda auditiva semelhante, cada criança, devido à possibilidade ou não de realizar o IC, também pôde apresentar diferenças nos questionários.

A criança da família PD4, do grupo 3, apresentou perda auditiva de 55 dBNA (média de 500 a 2 kHZ), o que mostra que, quanto menor a perda auditiva, melhores são as respostas de escores encontradas no IT MAIS e MUSS. Provavelmente, pelo grau da perda auditiva e comparecimento em terapia com mais de $70 \%$ de frequência $(72,7 \%)$, houve boa avaliação do grau de envolvimento familiar (avaliação 4) e também em relação ao uso do AASI durante seis horas por dia. O início da adaptação de AASI ocorreu quando a criança tinha 10 meses.

O envolvimento familiar (Tabela 2 e Figura 4), foi semelhante nos três grupos. Nas avaliações do grau de envolvimento familiar, realizadas pelas fonoaudiólogas que atendiam as crianças no ADAPTI, foi possível verificar dois extremos (Figura 4).

A família FC3 apresentou avaliação 2 na escala de envolvimento familiar, possivelmente por ter comparecido poucas vezes ao atendimento terapêutico $(54,5 \%)$, o que sugere pouco envolvimento da família no processo de reabilitação auditiva da criança.

Diferentemente, a família FC6 apesar da avaliação 2, compareceu a todos os atendimentos agendados (100\%), tanto nas intervenções terapêuticas quanto no GrAF. Portanto, a justificativa para a avaliação que recebeu da terapeuta não seria o comparecimento ao serviço, como no caso anterior. Sendo assim, provavelmente essa nota reflete o fato de a criança ter iniciado tardiamente a adaptação de AASI.

As crianças das famílias FC3 e FC6 chegaram para diagnóstico e intervenção fonoaudiológica após os dois anos de idade, com 35 e 34 meses respectivamente.

Outro extremo apresentado na Figura 4 refere-se à família PD5, que teve avaliação 5 na escala de avaliação de envolvimento familiar. Essa pontuação justifica-se, pois, assim que foi adaptada ao uso do AASI (quando tinha 2 meses), a criança começou, concomitantemente, o ADAPTI e o processo de avaliação para a cirurgia do IC. Os pais compareceram em 72,7\% dos atendimentos terapêuticos agendados, mostrando-se envolvidos em todo o tratamento proposto no Serviço de Saúde Auditiva.

Observa-se que os indivíduos do grupo 3 apresentaram tendência a usar o aparelho por mais horas do que os dos outros grupos (Tabela 3 e Figura 5).

O pouco uso diário do AASI (duas horas) pela criança da família FC6, mesmo comparecendo a todas as terapias e GrAF, pode estar relacionado ao fato que a Língua Brasileira de Sinais (LIBRAS) foi a escolha da família desde o princípio. Nesse caso, considerando pouca audibilidade de sons de fala, o uso do aparelho pode não ser percebido como fator que contribui para a comunicação, considerando que a LIBRAS foi se tornando a língua principal de comunicação da criança.

No Grupo 3 estão as famílias FC2, cuja criança usa o AASI oito horas por dia, e a família C3, cuja criança faz uso do dispositivo duas horas diárias.
O grupo que demonstrou maior envolvimento foi o de crianças que, em sua maioria, apresentaram média de perdas auditivas de 55dBNA (PD4), 65dBNA (C3) e 70dBNA (FC1), representando $75 \%$ (Figura 4). Nele, havia uma criança com média de perda auditiva de 90dBNA (FC2), porém com o maior tempo de uso do AASI (oito horas/dia). Vale ressaltar, que tratava-se de uma das crianças mais velhas do grupo, com adesão a $80 \%$ de frequência no atendimento terapêutico, cuja família, embora tenha tido classificação 3 no envolvimento familiar, pareceu, durante o GrAF, preocupada com as questões futuras da criança, no que diz respeito à audição, linguagem e escolaridade. As boas respostas nos questionários utilizados ocorreram pelo fato de se tratar de uma criança mais velha (iniciou a adaptação de AASI aos 37 meses de idade) e que, portanto, rapidamente adquiriu as habilidades questionadas nos instrumentos IT MAIS e MUSS, apresentando alta porcentagem nos escores de ambos os instrumentos.

Outra exceção nesse grupo diz respeito à criança C3, monitorada no Datalog e apresentando apenas duas horas de uso do AASI. Segundo a mãe, a criança não estava usando o AASI como anteriormente (com mais frequência), porque o irmão gêmeo estava muito doente, sobrecarregando a família, levando a mãe a colocar menos vezes o aparelho na criança. Nesse caso, embora seja uma mãe considerada participante, $o$ uso do AASI não parece ser valorizado pela família.

Observou-se que a maioria das mães dos três grupos participou do GrAF. A porcentagem dos pais que não participaram é maior ou igual a $50 \%$ nos três grupos, sendo que a menor porcentagem de participação foi verificada no grupo 2. É possível dizer que não houve diferença quanto ao fato de os pais terem ou não participado do GrAF PD ou FC, pois as frequências se assemelham (Tabela 4).

\section{DISCUSSÃO}

Como observado no presente estudo, a maior participação das mães no ADAPTI indica que elas passam grande parte do tempo com os filhos, sendo as responsáveis por acompanhá-los nos atendimentos realizados. Pelo fato de exercerem atividades profissionais, os pais são menos presentes nesse momento. Cabe ao fonoaudiólogo reconhecer a importância do pai na vida da criança e procurar inseri-lo cada vez mais no processo de (re) habilitação auditiva ${ }^{(16-18)}$.

A intervenção logo após o diagnóstico da criança deficiente auditiva é de extrema importância para que ocorra o desenvolvimento das habilidades auditivas, de fala e linguagem. Para isso, é fundamental o uso do AASI e/ou outros dispositivos, como o Implante Coclear ${ }^{(11,18-21)}$.

O fato das respostas ainda não serem consistentes nas etapas iniciais de adaptação de AASI, dependendo do grau de perda auditiva da criança, diminui a motivação dos pais em insistir no uso dos dispositivos. Conforme as respostas aparecem, criança e família sentem falta dos $\mathrm{AASI}^{(4)}$. 
Tabela 4. Frequências e porcentagens de "Participou de GrAF" (mãe) por grupo/Frequências e porcentagens de "Participou de GrAF" (pai) por grupo

\begin{tabular}{|c|c|c|c|c|c|c|}
\hline \multirow{2}{*}{ Grupo (Participação da mãe) } & \multicolumn{2}{|c|}{ Não } & \multicolumn{2}{|c|}{ Sim } & \multicolumn{2}{|c|}{ Total } \\
\hline & $\mathrm{n}$ & $\%$ & $\mathrm{n}$ & $\%$ & $\mathrm{n}$ & $\%$ \\
\hline 1 & 1 & 20,0 & 4 & 80,0 & 5 & 100,0 \\
\hline 2 & 1 & 20,0 & 4 & 80,0 & 5 & 100,0 \\
\hline 3 & 1 & 25,0 & 3 & 75,0 & 4 & 100,0 \\
\hline Total & 1 & 21,4 & 11 & 78,6 & 14 & 100,0 \\
\hline \multirow{2}{*}{ Grupo (Participação do pai) } & \multicolumn{2}{|c|}{ Não } & \multicolumn{2}{|c|}{ Sim } & \multicolumn{2}{|c|}{ Total } \\
\hline & $\mathrm{n}$ & $\%$ & $\mathrm{n}$ & $\%$ & $\mathrm{n}$ & $\%$ \\
\hline 1 & 3 & 60,0 & 2 & 40,0 & 5 & 100,0 \\
\hline 2 & 4 & 80,0 & 1 & 20,0 & 5 & 100,0 \\
\hline 3 & 2 & 50,0 & 2 & 50,0 & 4 & 100,0 \\
\hline Total & 9 & 64,3 & 5 & 35,7 & 14 & 100,0 \\
\hline
\end{tabular}

Neste estudo, a adesão ao uso do dispositivo eletrônico não esteve relacionada com significância estatística a nenhum fator específico, em virtude do número de sujeitos na amostra. No entanto, observou-se tendência de melhores repostas quanto à escala de avaliação do envolvimento familiar, uso do AASI e frequência nas terapias nas crianças que iniciaram antes o diagnóstico e a intervenção. A adesão ao tratamento é um processo multifatorial que se estrutura em uma parceria entre quem cuida e quem é cuidado. Diz respeito à frequência, à constância e à perseverança em busca da saúde ${ }^{(22)}$.

O envolvimento de algumas famílias no grupo de adesão e a participação nas atividades propostas sugere que esta pode ser uma estratégia com repercussões na adesão ao processo de reabilitação auditiva. De fato, envolver, acolher, criar parcerias e vínculos com os familiares durante o trabalho fonoaudiológico é fundamental para alcançar um prognóstico favorável e resultados satisfatórios em relação ao uso do AASI pelas crianças ${ }^{(5,23)}$.

A diversidade nas características das famílias deste estudo, nos grupos organizados de acordo com habilidades auditivas e de linguagem, sugere que é necessária atenção especial aos processos iniciais do atendimento terapêutico, com a realização de procedimentos de acolhimento das famílias para que a parceria entre profissional e família possa ser estabelecida, visando promover a utilização mais consistente do AASI nas situações cotidianas pelas crianças ${ }^{(23)}$. Quanto mais acesso ela tiver às informações transmitidas por meio dos contatos sociais estabelecidos, melhor será seu desenvolvimento de linguagem oral. Portanto, se o uso do AASI não é efetivo e constante, a criança perde informação auditiva, o que resulta em dificuldades de comunicação ${ }^{(24-27)}$.

Os profissionais envolvidos precisam orientar e aconselhar os pais, fornecendo informações claras sobre diagnóstico, uso do AASI, seus benefícios e limitações, manuseio, manutenção e cuidados para a utilização segura da prótese auditiva e progressos que a criança pode atingir com o aparelho e com o programa de intervenção. Nesse sentido, assegurar a adaptação e a utilização diária do AASI permite que as crianças tenham experiências com sua audição em todas as horas do dia, praticamente.

O fonoaudiólogo deve orientar os pais ou responsáveis pela criança sobre os controles do aparelho, sobre moldes auriculares, bateria e cuidados em relação ao manuseio e manutenção ${ }^{(27,28)}$. É importante adaptar o AASI na criança o mais cedo possível e também acompanhá-la em um serviço especializado $^{(11,19)}$. No entanto, nossa experiência com os grupos estudados sugere que a compreensão da família sobre a importância da reabilitação auditiva depende de estratégias de motivação e ajustes de expectativas, indo muito além da informação da importância do dispositivo eletrônico.

É importante destacar que, muitas vezes, a atitude positiva em relação aos processos de retornos agendados pode ser confundida com o que a escala de envolvimento familiar busca determinar. Daí a discrepância encontrada nas diferentes famílias avaliadas neste estudo.

\section{CONCLUSÃO}

O uso sistemático de AASI foi a única variável com forte relação com habilidades auditivas e de linguagem. Grau de perda, idade de início de amplificação e processo terapêutico com ou sem participação no Grupo de Adesão não explicaram o desenvolvimento das crianças do estudo. A baixa adesão ao uso sistemático dos AASI por parte de pais participativos nos GrAF sugere que objetivos específicos e de curto prazo, nessa direção, devem ser incluídos nas metas a serem atingidas pelos grupos.

Manifestações de envolvimento familiar, espelhadas na disponibilidade para a participação, nem sempre garantiram a utilização sistemática e consistente do AASI pela criança. Nesse sentido, são necessárias propostas de ações no âmbito da intervenção em Serviços de Saúde Auditiva que garantam a adesão ao tratamento, para que medidas de resultados possam refletir o investimento na audição residual. Esse fato sugere que, mesmo em famílias aparentemente comprometidas com a intervenção, é imprescindível a verificação do número de 
horas utilizadas por dia, por meio de recursos objetivos, como o Datalog no AASI.

No caso de perdas auditivas profundas, quanto antes forem realizados exames complementares para encaminhamento a cirurgia de Implante Coclear, mais preciso será o posicionamento dos profissionais quanto ao prognóstico para oralidade e, consequentemente, para as adaptações às expectativas da família.

Outros estudos são necessários, envolvendo monitoramento de resultados de desenvolvimento auditivo e de linguagem e características de adesão ao programa de intervenção na população atendida em Serviços de Saúde Auditiva.

\section{REFERÊNCIAS}

1. Moeller MP. Early Intervention and language development in children who are deaf and hard of hearing. Pediatrics. 2000;106(3):43-7.

2. Yoshinaga-Itano C. From screening to early identification and intervention: discovering predictors to successful outcomes for children with significant hearing loss. J. Deaf Stud. Deaf Educ (Oxford). 2003;8(1):11-30.

3. Rissatto MR, Novaes BCAC. Hearing aids in children: the importance of the verification and validation process. Pro Fono. 2009;21(1):131-6.

4. Novaes BCAC, Mendes BCA. Terapia fonoaudiológica da criança surda. In: Fernades FDM, Mendes BCA, Navas ALPGP. (Orgs). Tratado de Fonoaudiologia. 2a.ed. São Paulo: Roca; 2009. p. 202-9.

5. Sininger YS, Grimes A, Christensen E. Auditory development in early amplified children: factors influencing auditory-based communication outcomes in children with hearing loss. Ear Hear. 2010;31(2):166-85.

6. Muñoz K, Roberts M, Mullings D, Harward R. Parent hearing aid experiences. Volta Review. 2012;112(1):63-76.

7. Hoffman J, Beauchaine K. Babies with hearing loss: steps for effective intervention. ASHA Leader. 2007;12(2):8-23.

8. Figueiredo RSL, Novaes, BCAC. Rumo às primeiras palavras: o enquadre na terapia fonoaudiológica do bebê com deficiência auditiva. Rev CEFAC. 2012;5(1):50-8.

9. Nery DM, Novaes BC. Identificação de estratégias no processo terapêutico de uma criança deficiente auditiva. Distúrb Comum. 2001;13(1):49-67.

10. Scappaticci ALSS. A importância do sistema familiar no desenvolvimento da criança: um enfoque para a Pediatria. In: Carvalho ES, Carvalho WB. Terapêutica e prática pediátrica. 2a ed. São Paulo: Atheneu; 2001. p. 73-7.

11. Halpin KS, Smith KY, Widen JE, Chertoff ME. Effects of universal newborn hearing screening on an early intervention program for children with hearing loss, birth to $3 \mathrm{yr}$ of age. J Am Acad Audiol. 2010;21(1):169-75.
12. Novaes BCAC. Hearing impaired children in São Paulo, Brazil: knowledge and attitudes of mothers regarding hearing impairment and early intervention programs, and the implications for habilitation. Columbia: Columbia University; 1986.

13. Robbins AM, Osberger MJ. Meaningful use of speech scales. Indianápolis: University of Indiana School of Medicine; 1990.

14. Zimmerman-Philips S, Osberger MJ, Robbins AM. Infant-Toddler: meaningful auditory integration scale (IT-MAIS). Sylmar, Advanced Bionics Corporation; 1997.

15. Johnson RA, Wichern DM. Applied Multivariate Statistical Analysis. 3rd ed. New Jersey: Prentice-Hall; 1992.

16. Boscolo CC, Momensohn-Santos TM. A deficiência auditiva e a família: sentimentos e expectativas de um grupo de pais de crianças com deficiência da audição. Distúrb Comum. 2005;17(1):69-75.

17. Caldas FF, Lemos AB, Tschiedel RS. O envolvimento do pai no processo de (re) habilitação auditiva de seu filho deficiente auditivo. Comum Ciênc Saúde. 2009;20(1):17-28.

18. Erenberg A, Lemons J, Sia C, Trunkel D, Ziring P. Newborn and infant hearing loss: detection and intervention, American Academy of Pediatrics. Task Force on Newborn and Infant Hearing, 1998 1999. Pediatrics. 1999;103(2):527-30.

19. Harisson M, Rouch J, Wallace J. Trends in age of identification and intervention in infants with hearing loss. Ear Hear. 2003;24(1):89-95.

20. Gatto CI, Tochetto TM. Deficiência auditiva infantil: implicações e soluções. Rev CEFAC. 2007;9(1):110-5.

21. Prendergast S, Lartz MN, Fiedler BC. Ages of diagnosis, amplification, and early intervention of infants and young children with hearing loss: findings from parent interviews. Am Ann Deaf. 2002;147(1):24-30.

22. Silveira LMC, Ribeiro VMB. Grupo de adesão ao tratamento: espaço de "ensinagem" para profissionais de saúde e pacientes. Interface. 2005;9(16):91-104.

23. Moeller MP, Hoover B, Peterson B, Stelmachowicz P. Consistency of hearing aid use in infants with early-identified hearing loss. Am J Audiol. 2009;18(1):14-23.

24. Serbetcioglu MB. Critical learning period for speech acquisition and screening techniques in early detection of hearing impairment. Turk J Pediatr. 2001;43(2):128-32.

25. Moeller MP. Optimizing early word learning in infants with hearing loss. Audiology Today. 2010;18(1):19-27.

26. Fagan MK, Pisoni DB. Hearing experiences and receptive vocabulary development in deaf children with cochlear implants. J Deaf Stud Deaf Educ. 2010;15(2):149-61.

27. Brazorotto JS. A terapia fonoaudiológica da criança surda. In: Bevilacqua MC, Moret ALM. Deficiência Auditiva: Conversando com familiares e profissionais de saúde. São José dos Campos: Pulso; 2005. p. 203-23.

28. Sjoblad S, Harrison M, Roush J, McWilliam RA. Parent's reactions and recommendations after diagnosis and hearing aid fitting. Am J Audiol. 2001;10(1):24-31. 